\title{
The Design and Implementation of a Performance Evaluation Tool with TPC-W Benchmark
}

\author{
Zhu Hong ${ }^{1}, \mathrm{Fu} \mathrm{Xin}^{1}$, Lin Qiu Hui ${ }^{1}$ and Kevin Lü ${ }^{2}$ \\ ${ }^{1}$ Huazhong University of Science and Technology, Wuhan, Hubei, China \\ ${ }^{2}$ Brunel University, Uxbridge, UK
}

\begin{abstract}
This paper describes the design and implementation of a performance testing tool for E-commerce systems. This tool can be used to assess the system performance based on TPC-W benchmark. The main features and designing considerations of the tool as well as testing procedures are discussed. The test results of applying this tool to two real systems have been initially used for system analysis and evaluation.
\end{abstract}

Keywords: TPC-W, testing tool, performance benchmark.

\section{Introduction}

Performance evaluation for E-commerce applications has become an important issue. It can be used to compare different systems, to determine a suitable configuration for an application, to tune its performance or to decide how to upgrade it. Performance evaluation tools that automate the process of comparing and evaluating design decision would benefit those involved with application sizing, system design and administration.

A typica 1 E-commerce application system consists of Web server, back-end database server and network components. Its performance depends on these components. A number of benchmarks have been proposed and used for Ecommerce applications so far [9] [11]. Amongst these benchmarks, TPC-W was developed specifically for E-commence systems, which has been widely recognized and accepted.

Several testing systems using TPC-W benchmark for evaluating systems have been developed $[7,9]$. Harold W. Cain et al [1] at Wiscon- sin University developed a Java TPC-W evaluation system. The system has three tiers in architecture and its design and implementation are in accordance with TPC-W specification, except the secure socket layer required for the Purchase confirm interaction. In this system, as the SSL is used [6], the Web server will do additional work for the purchase-confirm-interactions besides the one required by the specification and the performance of the whole system will be decreased largely [5]. Daniel F. et al addressed the architecture and model of the testing system based on TPC-W benchmark and implemented a testing system with PHP technology. The metrics of their evaluation are: the web interaction per seconds (WIPS) [5]. Pierfrancesco Foglia et al [3] [4] studied the workload based on TPC$\mathrm{W}$ benchmark for E-commerce and designed a testing system with three tiers architecture to evaluate the memory performance of SMP multiprocessors running on the workload produced by the TPC-W testing system. Most of the existing testing systems were designed to evaluate the performance of server hardware and few discussed the performance evaluation involving other components of the Web servers.

This paper deals with the design and development of a performance evaluation tool using TPC-W benchmark for E-commerce applications. This test tool is capable of assessing different components of E-commence applications systems including server hardware, web server software, database server and communication facilities. Throughput is the main factor 
used to measure system performance. The accessorial statistics of the resources utilization, such as the CPU utilization, memory utilization and $\mathrm{I} / \mathrm{O}$ activities have been used to analyze the bottleneck of performance. This test tool has been used to comparing DM4 [8] and SQL Server 2000 [10]. The test results have been used for system design analyses.

The general performance metrics are introduced in Section 2. Section 3 briefly describes the architecture of the test tool. The main features of TPC-W are summarized in Section 4. Section 5 provides the implementation details of the testing tool. Section 6 presents some test results and initial analyses. Section 7 presents the summary.

\section{The Performance Metrics}

TPC-W emulates a bookstore on the Internet. It comprises a set of basic operations designed to exercise transactional Web system in a manner representative of Internet commerce application environments. The activities of users are browsing, searching and ordering. It defines eight database basic related tables (see Figure 1) and 14 Web interactions (see Table 1). These Web interactions include two types: browsing and ordering. And these Web interactions must satisfy the ACID properties.

The primary metric of the TPC-W benchmark is a throughput which consists of two main metrics. The first is denoted as WIPS @ SF, which is the average number of Web interactions per second completed during Shopping Interval where about $80 \%$ of the Web interactions are browsing and $20 \%$ of the Web interactions are ordering. The second is price performance metric defined as the total cost of the SUT divided by the number of WIPS measured in Shopping Interval. In the mean time, there are two additional metrics for throughput. One is the WIPSb, which is the average number of Web interactions per second completed during the Browsing Interval. Among all the Web interactions, 95\% of them are browsing (bookstore home page, new product and best seller, product detail and searching) while $5 \%$ of the Web interaction transactions are ordering. The metric WIPSb reflects the query performance of DBMS. The other is the WIPSo, which means the average number of Web interactions per second completed during the Ordering Interval and about 50\% of the Web interaction transactions are ordering and shopping respectively. This metric reflects the data updating performance in database server. Among these metrics, the Web interactions response time must satisfy the limitations specified by TPC-W.

In addition, the resources which need to be monitored and analyzed include CPU utilization, memory utilization and I/O activities to provide additional information for the benchmark customer or developer of DBMSs. The CPU utilization is the percentage of time for which the CPU is busy and it reflects the potentiality of a database server on data processing. The memory utilization indicates how the DBMS supports the demands of the TPC-W benchmark efficiently and whether the DBMS have the ability to improve its performance on memory utilization. The $\mathrm{I} / \mathrm{O}$ activities in database indicate the ability of the DBMS dealing with I/O operations and if there are still rooms for performance improvement.

\section{The Architecture of the Test Tool}

The framework of a typical E-commerce application can be logically described as a three-tier computing model. In order to test the performance of E-commerce system, the testing system should produce workload close to real applications. By measuring the number of Web interactions per second, we will be able to evaluate the ability of the DBMS on processing transactions from network and the performance of whole system under test (SUT).

The test tool system is designed as three-tier architecture, shown in Fig. 1. The first tier is the one shown in the dashed box on the left. It consists of several client computers; each of them emulates a number of Web browsers and requests of the service provided by the system under test. In order to display the performance of the whole system during a test interval, the Remote Browser Emulators (RBEs) are divided into two types: there is one master RBE and several secondary RBEs. During a test interval, the master $\mathrm{RBE}$ receives the test results from 


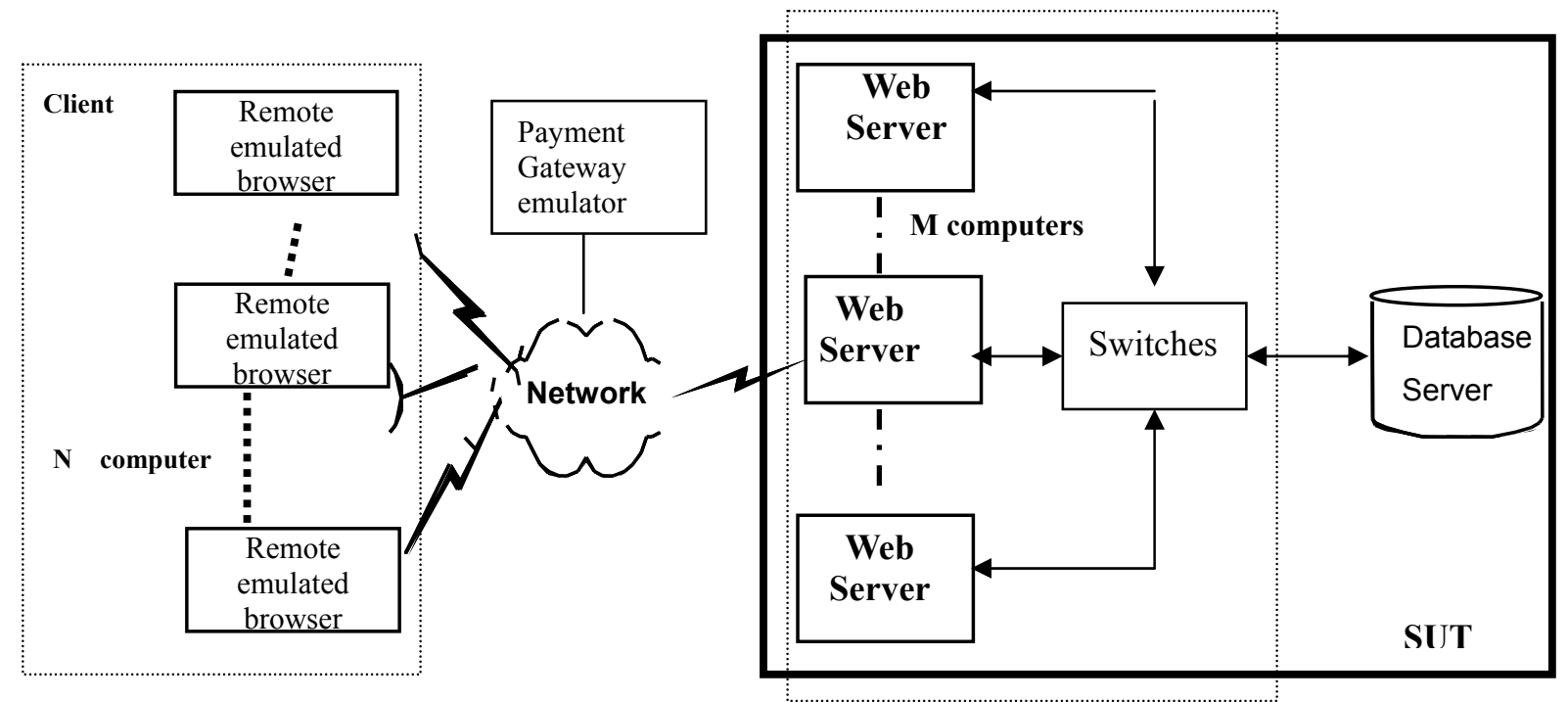

Fig. 1. The architecture of the test tool.

the secondary RBEs to display the performance metrics of SUT. At the same time the master $\mathrm{RBE}$ also emulates browsers and produces the workload for SUT. The master RBE creates a thread to implement this function. The second tier is the one shown in the dashed box on the right and consists of several Web servers. They compose a cluster of Web servers to balance the workload from the RBEs. The third-tier is the database server on the right.

According to the TPC-W specification, the system under test consists of a number of application software packages, such as Web server, database server, but except payment gateway emulator. As the payment gateway emulator will consider the processes of the purchase confirm interaction, and therefore its cost will not be included in the cost of SUT. Web servers are the connection to the RBEs, and all the Web interactions begin with the request to Web server from RBEs. The Web server sends requests to database server and payment gateway, and navigates RBEs to receive html pictures and images from Web server to response the requests.

The testing system architecture is designed according to the typical features of the E-commerce applications. This testing system not only can be used to test the performance of E-commerce applications, but can also be used to evaluate the performance of database management systems or Web servers.

\section{Data Sets in Web Interaction Transaction}

\subsection{Tables in Database}

The TPC-W specification defines eight tables for the database. We add another two tables for cart information as accessorial table. All of the 10 tables and their relationships are defined in the entity-relationship diagram shown in Fig. 2. Dotted lines in Fig. 2 represent one-toone relationships between non-key fields related through a business rule. These fields are shown in italic. The arrows point in the direction of one-to-many relationships between tables. And the bold types identify primary and foreign keys. According to TPC-W specification, additional tables may be added to describe cart information. All the extensions are permitted.

\subsection{The Database Scaling}

Database scaling is defined by the cardinality (number of rows) of the ITEM table and the number of EB's configured for WIPS. The cardinality of the ITEM table, the number of row in the ITEM table, must be chosen from the set of defined scale factors: 1,$000 ; 10,000 ; 100,000$; $1,000,000 ; 10,000,000$. The tester must select 


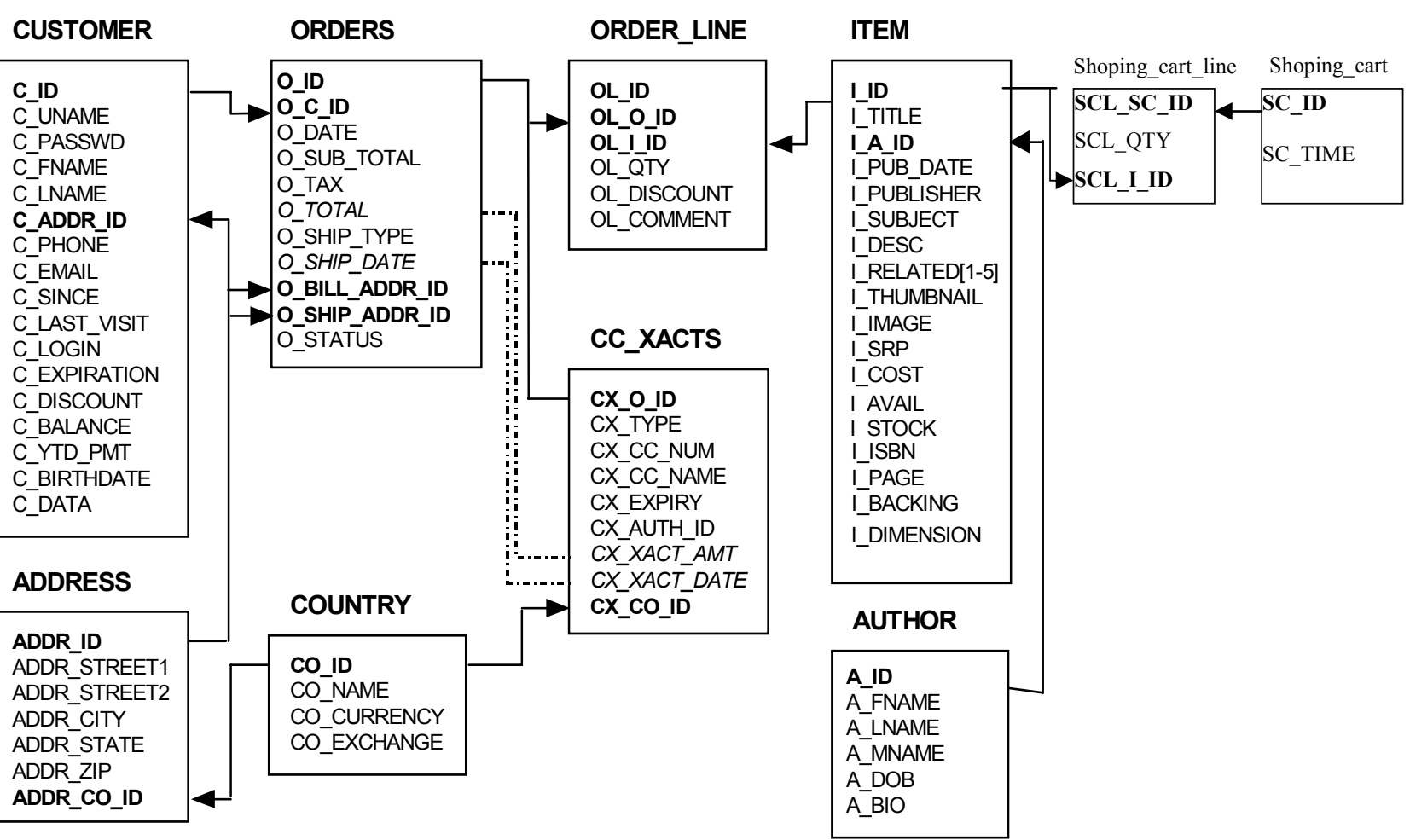

Fig. 2. The relationships between tables in the database.

\begin{tabular}{|l|l|l|l|}
\hline Table name & Cardinality (in rows) & Typical row length(bytes) & Typical table size(bytes) \\
\hline CUSTOMER & $2880 *$ (number of EB) & 760 & $2,188,888 \mathrm{k}$ \\
\hline COUNTRY & 92 & 70 & $6.44 \mathrm{k}$ \\
\hline ADDRESS & $2 *$ CUSTOMER & 154 & $887,040 \mathrm{k}$ \\
\hline ORDERS & $0.9 *$ CUSTOMER & 220 & $570,240 \mathrm{k}$ \\
\hline ORDER_LINE & $3 *$ ORDERS & 132 & $1,026,432 \mathrm{k}$ \\
\hline AUTHOR & $0.25 *$ ITEM & 630 & $1,575 \mathrm{k}$ \\
\hline CC_XACTS & $1 *$ ORDERS & 80 & $207,360 \mathrm{k}$ \\
\hline ITEM & $1 \mathrm{k}, 10 \mathrm{k}, 100 \mathrm{k}, 1 \mathrm{M}, 10 \mathrm{M}$ & 860 & $8,600 \mathrm{k}$ \\
\hline Shoping_cart_line & & 12 & \\
\hline Shoping_cart & & 12 & \\
\hline
\end{tabular}

Note 1: Table sizes are computed for 1,000 EB's and 10,000 items

Note 2: Values for the ITEM table do not include the item's image and thumbnail

Note 3: The typical row lengths and table sizes given above are examples of what could result from an implementation. They are not requirements. They do not include storage and access overheads. The symbol " $\mathrm{k}$ " means one thousand and "M" means one million.

Note 4: No variation is allowed on table cardinality except on ORDER_LINE where the cardinality will vary slightly due to the random number of rows. Cardinality must meet a minimum requirement of 2.95 times the number of rows in the ORDER table.

Table 1. Database scaling rules after initialization.

a scale factor from these series to determine the database population. The cardinalities of these tables are shown as Table 1.

The cardinality of table AUTHOR (to store author information) is the function of ITEM from above table. The cardinality of table COUN-
TRY (to store country information) is fixed. The original cardinality of others is the function of the numbers of EB. Additionally, database population can't be changed for the secondary metrics WIPSo and WIPSb, but the number of EBs can be changed for them. The throughput 
of WIPS must satisfy the following inequation:

$$
\begin{gathered}
(\text { number of EB's }) / 14<\text { WIPS } \\
<(\text { number of EB's }) / 7
\end{gathered}
$$

The intent of this inequation is to prevent reporting a throughput that exceeds the maximum, where the maximum throughput is achieved with infinitely fast Web interactions resulting in a null response time and minimum required think times (think time is the time elapsed from the last byte received by the EB to complete a Web interaction until the first byte sent by the EB to request the next Web interaction). It emulates the time the user hit the Web pages so that the test tool emulates the real environment. This is computed to be $1 / 7$ WIPS per EB. To prevent over-scaling the SUT, the throughput cannot fall short of the above $1 / 14$ WIPS per EB, which represents $50 \%$ of the computed maximum throughput.

\subsection{Web Interaction Transactions}

TPC-W defines 14 Web interactions simulated from a typical Internet business environment. These transactions involve searching, updating, browsing. They are divided into two types: browsing and ordering. Most of these browsing transactions are database query based while most ordering transactions involving operations of insert, update and delete operations on database. All of the 14 transactions satisfy basic ACID transaction properties: atomicity, consistency, isolation and durability. The complexity of these 14 transactions accessing database is different.

The customer's behaviors are divided into three types: browsing, shopping and ordering according to their purposes. Different customers have different ratios of Web interactions. The ratios of the Web interaction transactions in three

\begin{tabular}{|l|c|c|c|}
\hline \multicolumn{1}{|c|}{ Web Interaction } & Browsing Mix (WIPSb) & Shopping Mix (WIPS) & Ordering Mix (WIPSo) \\
\hline Browse & $\mathbf{9 5} \%$ & $\mathbf{8 0} \%$ & $\mathbf{5 0} \%$ \\
\hline Home & $29.00 \%$ & $16.00 \%$ & $9.12 \%$ \\
\hline New Products & $11.00 \%$ & $5.00 \%$ & $0.46 \%$ \\
\hline Best Sellers & $11.00 \%$ & $5.00 \%$ & $0.46 \%$ \\
\hline Product Detail & $21.00 \%$ & $17.00 \%$ & $12.35 \%$ \\
\hline Search Request & $12.00 \%$ & $20.00 \%$ & $14.53 \%$ \\
\hline Search Results & $11.00 \%$ & $17.00 \%$ & $13.08 \%$ \\
\hline Order & $\mathbf{5} \%$ & $\mathbf{2 0} \%$ & $\mathbf{5 0} \%$ \\
\hline Shopping Cart & $2.00 \%$ & $11.60 \%$ & $13.53 \%$ \\
\hline Customer Registration & $0.82 \%$ & $3.00 \%$ & $12.86 \%$ \\
\hline Buy Request & $0.75 \%$ & $2.60 \%$ & $12.73 \%$ \\
\hline Buy Confirm & $0.69 \%$ & $1.20 \%$ & $10.18 \%$ \\
\hline Order Inquiry & $0.30 \%$ & $0.75 \%$ & $0.25 \%$ \\
\hline Order Display & $0.25 \%$ & $0.66 \%$ & $0.22 \%$ \\
\hline Admin Request & $0.10 \%$ & $0.10 \%$ & $0.12 \%$ \\
\hline Admin Confirm & $0.09 \%$ & $0.09 \%$ & $0.11 \%$ \\
\hline
\end{tabular}




\begin{tabular}{|l|c|c|c|c|}
\hline Page name & Dynamic & Join tables & Images & $\begin{array}{c}90 \% \text { response time } \\
\text { limitation (seconds) }\end{array}$ \\
\hline Admin Confirm & Y & 4 & 5 & 20 \\
\hline Admin request & Y & 2 & 6 & 3 \\
\hline Best Seller & Y & 4 & 9 & 5 \\
\hline Purchase Confirm & Y & 7 & 2 & 3 \\
\hline Purchase Request & Y & 6 & 3 & 3 \\
\hline Customer Registration & N & NO & 4 & 3 \\
\hline Home & Y & 2 & 9 & 3 \\
\hline New Product & Y & 2 & 9 & 3 \\
\hline Order Display & Y & 5 & 2 & 3 \\
\hline Order Inquiry & Y & 1 & 3 & 3 \\
\hline Product Detail & Y & 2 & 6 & 10 \\
\hline Search Request & Y & 1 & 9 & 3 \\
\hline Search Result & Y & 2 & 9 & 9 \\
\hline Shopping Cart & Y & 3 & & 9 \\
\hline
\end{tabular}

Table 3. Typical features of 14 Web interaction transactions.

types of working mode are all listed in Table 2. At the same time, different Web transactions have different dynamic characterizations. The number of the table, network object and response time are different. Table 3 lists each typical Web transaction; the number of tables join involved additional base tables for describing the carts.

\section{Implementation}

There are two main issues needing to be resolved: (1) Remote emulated browser (RBE) initiates Web interaction transactions; (2) credit card authorization associated with some Web interactions.

\subsection{Implementation of the RBEs}

The RBEs simulate the users who use browser access to the system under test. A RBE creates and manages a thread for each emulated browser respectively. The testing system emulates the scenario in which large numbers of users concurrently request services and these users have different types of Web interactions. We use Java multi-thread technology to create large numbers of users emulate Web interactions and requests. At the same time, the system will record a number of performance parameters, such as: type of transaction, quantity, response-time. Fig. 3 shows the operational procedure of the RBEs.

Besides, we create another two types of threads in RBEs. When a RBE is configured as a master client (only one master client in the whole testing system), it creates a thread to receive test data from other secondary clients. At the same time, the master RBE will combine all the data from secondary clients and display the metrics for the whole SUT. While the RBE is configured as secondary client, it sends the test data during testing interval to the master client. Although running each one of the two types of threads will consume more CPU time, especially the master client receives data from several secondary clients. But we can configure less number of 


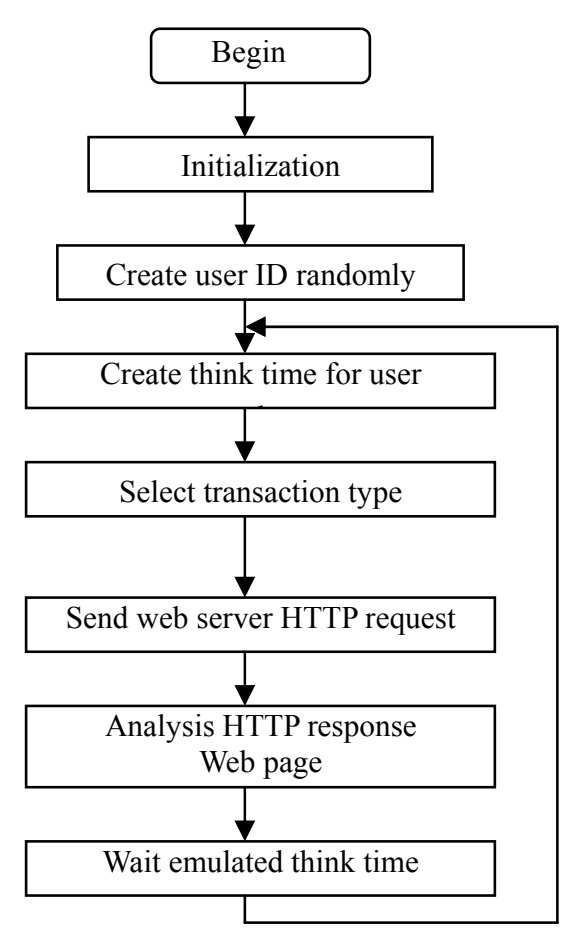

Fig. 3. The operational procedure of the RBEs.

the EBs for the master client. This communication workload would decrease. That will not affect the overall workload and the results of the whole SUT.

\subsection{The Credit Card Authorization}

Secure socket layer is a secure communication protocol released by Netscape. SSL uses public-key cryptography to authenticate connected participants and to make a private key used to encrypt data sent over the SSL connection. SSL authentication uses a certificate, made up of a public key and some information about the owner of the corresponding private key. The procedure of credit card authorization is:

(1) The customer provides URL of Web server, name, password and location of private key.

(2) The customer sends a Purchase request to Web server.

(3) Web server gets the certificate from PGE, and verified the validity of the certificate using the public key of PGE. Then get the public key after passing the verification.
(4) The Web server creates a session key for communication and encrypts it with public key.

(5) The customer will get the public key of Web server from PGE in the same way.

(6) After the customer decrypted the session key with his private key, he will encrypt the public key with session key and send it back to Web server.

(7) The server decrypts the session key with his private key, compares the session key with the original session key the user sent. If they are the same, the authentication is passed, otherwise authentication is failed.

The Web server will communicate with the customer using the session key to encrypt data. Although the authentication does not consume much resource, this procedure will require communication time and affect the response time. From the test results in paper [5], the WIPS is about $15 \%$ lower than that without SSL communications.

\subsection{Modules}

\subsubsection{Data Generation Tool}

Data generation tool is designed to generate a large amount of data close to real application scenarios in short period of time. There are two ways for data generation. One is based on the number of EBs and rows in ITEM table to generate data randomly, the other one is to transfer data from one database to another to maintain the same data in different databases. We use the Java Database Connection (JDBC) API to connect databases. In order to speed up the data loading, we use the pre-prepared statements in JDBC which bind the data to every column of each base table.

\subsubsection{ACID Properties Test Module}

By the TPC-W specification, the database server must ensure that the DBMS satisfying the ACID requirements. The purpose of these tests is to make sure that the ACID properties are applied in business environment. 


\subsubsection{Web Page Consistency Test Module}

Web pages returned by the SUT to the EB reflect the content of the database through displaying plain data and Web objects (GIF pictures). As database transactions update the content of the database, the Web pages must display a consistent reflection of these updates, with the exception of the search result pages for title and author searches. This module emulates users' activities to test the SUT if it reaches Web page consistency.

\subsubsection{Performance Test Module}

We will acquire the data and store it in database for the performance metrics for each DBMS: Web interaction transaction per second (WIPS), the price of per Web interaction (\$/WIPS), Web interaction transaction per second in browsing (WIPSb), Web interaction transaction per second in ordering (WIPSo), type, number, response time, ratio of success, mean think time of the interaction.

\subsubsection{Result Analysis Module}

The module will build graphs for the test procedure such as WIPS, WIPSb, WIPSo, and response time for every test. And it will display tables for 14 Web interaction transactions. The metrics include: WIPS, WIPSb, WIPSo, the number of users, start, interval and end of testing, mean think time and so on.

\section{Experimental and Results}

This test tool has been used to compare DM4 [8] and SQL Server 2000 [10]. Analyses of the testing procedure and of the initial testing results are introduced in this section.

\subsection{The Testing Procedure}

The final results of WIPS, WIPSb, WIPSo are the maximum processing ability of the system to research under the condition of response time limitation specified in Table 3.
The tests are performed following the procedure below:

(1) Load test data, specify the number of EBs;

(2) Fast test;

(3) Checking if the results will satisfy the requirements of the response time and think time. There are three options:

1. If they do not, it means that the workload is too heavy (too many users), and go to step 4;

2. If the response time is much less than the limitation of TPC-W specification, go to step 5;

3. If the workload is suitable, go to step 6;

(4) Decrease the number of EB, restore the data to initialization, go to step 2;

(5) Increase the number of EB, restore the data to initialization, go to step 2 ;

(6) Begin formal test, restore the data to initialization, test under the three modes respectively, end.

In the course of the tests above, the time intervals in formal test sustain 30 minutes. Before the test, the stabilization time needed for DBMS under the workload is 10 to 15 minutes. In the mean time, we can design optimize policy for the databases by creating indices or cluster indices on the base tables. These are permitted by TPC-W specification. We can configure the DBMS to reach optimal performance without changing the population of database during the test.

\subsection{Test Result and Analysis}

\subsubsection{The Testing Environment}

The test tool has been used to compare DM4 and Microsoft SQL Server 2000 respectively in the same hardware and software environments. The main difference between two SUTs is that they have different databases. The test environment is shown as follows: 


\begin{tabular}{|l|l|l|l|}
\hline Client & Web server & Database server \\
\hline OS & WINDOWS 2000 ADVANCED SERVER & WINDOWS 2000 ADVANCED SERVER & WINDOWS 2000 ADVANCED SERVER \\
\hline database & No & No & Microsoft SQL Server 2000 or DM4 \\
\hline Test system & TPC-W test system & Weblogic server & No \\
\hline CPU & P4 2. OG $\times 2$ computer & P4 2. $6 \mathrm{G} \times 2$ computer & $2 \times$ P4 2. 0G (Xeon) \\
\hline system & compatible computer & compatible computer & Legend T630 \\
\hline Memory & $2 \times 512 \mathrm{M}$ & $2 \times 512 \mathrm{M}$ & $2 \times 512 \mathrm{M}$ \\
\hline Disk & $1 \times 40 \mathrm{G} \mathrm{7200} \mathrm{turn}$ & $2 \times 80 \mathrm{G} \mathrm{7200} \mathrm{turn} \mathrm{(RAID} \mathrm{0)}$ & SCSI: $1 \times 72 \mathrm{G} \mathrm{10000} \mathrm{turn}$ \\
\hline Network card & $100 M b p s$ & $100 \mathrm{Mbps}$ & $100 \mathrm{Mbps}$ \\
\hline others & Symantec antivirus installed & Symantec antivirus installed & Symantec antivirus insta11ed \\
\hline
\end{tabular}

Table 4. The environments of software and hardware of two DBMS tested.

\subsubsection{Data Loading Test}

Before the test, we create table space of the capacity $25 \mathrm{G}$ for DM4 so that the system avoids unnecessary extension table space to speed up loading. We create initial base tables on the table space. At the same time we create a database of the capacity $25 \mathrm{G}$ for Microsoft SQL Server 2000. In the environment introduced in Table 4 , we tested the load time used, memory utilization, and CPU utilization in Table 5. The two databases run under their default configurations when the data is loaded. The test tool uses preprepared statement in JDBC to speed up loading and commits once after inserting 10000 rows.

The utilization of memory is an approximate range as we record them from tools provided by Windows 2000. Data loading time by DM4 is much slower than Microsoft SQL Server 2000, which indicates the performance of data manip- ulating under large quantities of data. As the memory utilization of DM4 is much less than Microsoft SQL Server 2000, it shows that DM4 has potential for improvement.

\subsubsection{Performance Test}

We tested the performance of DM4 and Microsoft SQL Server 2000 using the procedure introduced in section 6.1. The results are shown in Table 6. The curves shown in Fig 4 and 5, illustrate the metrics WIPS of Microsoft SQL Server 2000 and DM4. From this diagram we can see that Microsoft SQL Server 2000 has better performance than DM4, it runs very smoothly and doesn't fluctuate rapidly. Compared with Fig. 4 and Fig. 5, because the curve fluctuates rapidly, this indicates that there exists some performance bottleneck in DM4. After we compare WIPSb in Table 6 under the browsing mix ulteriorly

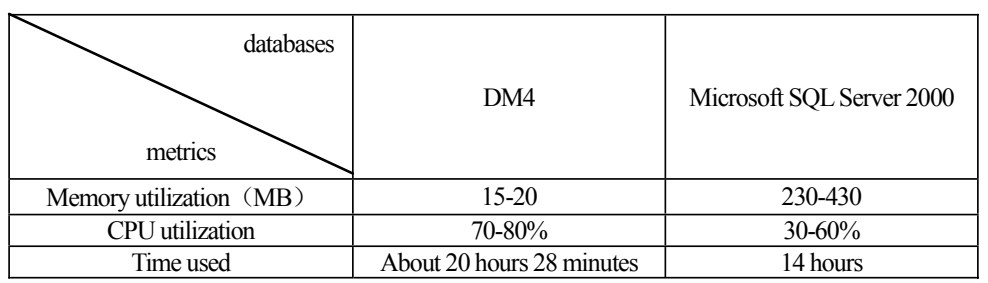

Table 5. The time consumed and CPU utilization in data loading test

\begin{tabular}{|c|c|c|c|c|c|c|c|c|c|c|}
\hline \multicolumn{5}{|c|}{ Microsoft SQL Server 2000} & \multicolumn{6}{|c|}{ DM4 } \\
\hline WIPS@10000: 443 & \multicolumn{2}{|c|}{ WIPSo@10000: 573} & \multicolumn{2}{|l|}{ WIPSb@10000: 355} & \multicolumn{2}{|c|}{ WIPS@10000: 286} & \multicolumn{2}{|c|}{ WIPSo@10000: 432} & \multicolumn{2}{|c|}{ WIPSb@10000: 203} \\
\hline \multicolumn{11}{|c|}{ Summary in } \\
\hline $\begin{array}{l}\text { Web } \\
\text { interaction }\end{array}$ & $\begin{array}{l}\text { Begin } \\
\text { time }\end{array}$ & $\begin{array}{l}\text { Interval of } \\
\text { test }\end{array}$ & $\begin{array}{l}\text { Mean } \\
\text { thinking time }\end{array}$ & $\begin{array}{l}\text { Number } \\
\text { of } \\
\text { users }\end{array}$ & $\begin{array}{l}\text { Begin } \\
\text { time }\end{array}$ & $\begin{array}{l}\text { Interva } \\
\text { test }\end{array}$ & of & $\begin{array}{l}\text { Mean } \\
\text { time }\end{array}$ & thinking & $\begin{array}{l}\text { Number of } \\
\text { users }\end{array}$ \\
\hline WIPS & $800 \mathrm{~S}$ & $1800 \mathrm{~S}$ & 6.96 & 3200 & $800 \mathrm{~S}$ & $1800 \mathrm{~S}$ & & 6.97 & & 2100 \\
\hline WIPSo & $1100 \mathrm{~S}$ & $1800 \mathrm{~S}$ & 6.98 & 4400 & 1100 & 1800 & & 6.96 & & 3200 \\
\hline WIPSB & 650 & 1800 & 7.03 & 2600 & 650 & 1800 & & 6.92 & & 1500 \\
\hline
\end{tabular}




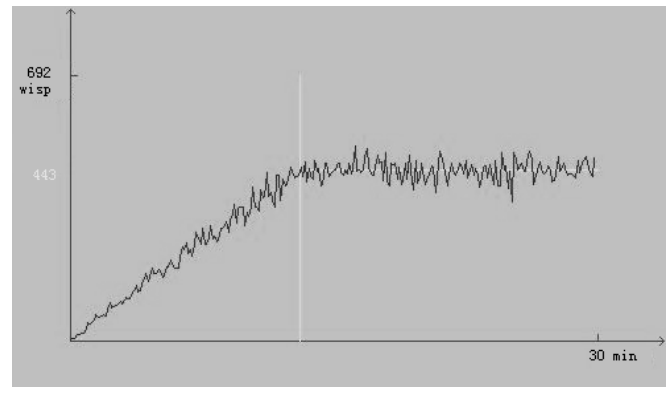

Fig. 4. Microsoft SQL Server 2000 wips.

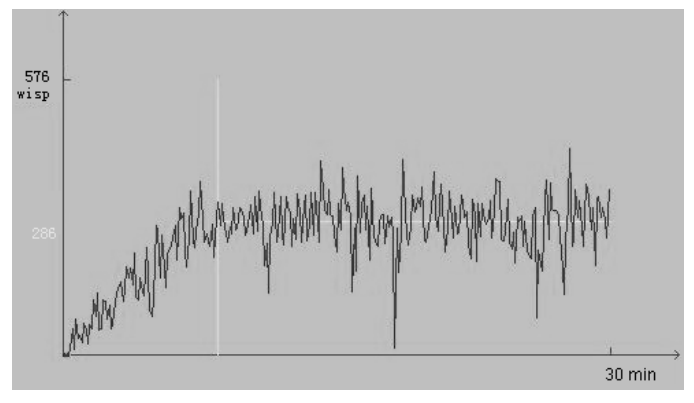

Fig. 5. DM4 wips.

of two DBMSs, we found that the query optimization needs to be improved in DM4, because most of the users (95\% with TPC-W limitation) in browsing mix mode are database queries and the ratio of data updating among users is $5 \%$. After we compare with WIPSo in Table 6, we can conclude that WIPSo in DM4 is a little better. It indicates that when users update small amount of data the performance in DM4 is fairly good, but still has a small gap with Microsoft SQL Server 2000.

Based on our initial test results of comparing DM4 and SQL Server 2000, we identified the following factors which affect the performance of DM4:

(1) Query optimization: because the WIPSb metrics of DM4 is low, one possible area needs to be improved is the query optimization policy. When the number of users increases, the number of tuples in database being locked will also increase, and the performance will decrease rapidly. Therefore, many requests will not get the response and be interrupted. Besides, TPC-W specifies some complex queries, so DBMS should provide different query optimization policies for them. But in DM4, it is required to give a special optimization SQL statement to perform this task in order to let the system reach its optimal performance, while Microsoft SQL Server 2000 doesn't need to regulate that, it can chose a suitable one automatically.

(2) Updating large quantity of data: The process of updating a large amount of data in DM4 can be very slow compared with Microsoft SQL Server 2000, especially for data loading meanwhile creating indexes. That indicates that the buffer management and memory utilization need to be investigated.

\section{Summary}

We have designed and implemented a testing tool running TPC-W. It is capable of measuring the system throughput and other performance metrics, as well as of collecting the accessory statistics of resources utilization, such as the CPU utilization, memory utilization and I/O activities. This tool can be been used to analyse various performance-related issues. By using this test system, we compared the performance of DM4 and Microsoft SQL Server 2000, and the initial test results have been used for DM4 performance analysis.

\section{References}

[1] H. W. Cain, R. Rajwar, M. Marden, M. H. LIPASTI, An architectural evaluation of Java TPC-W, in Proceedings of the Seventh International Symposium on High-Performance Computer Architecture (HPCA'01), NW Washington, DC USA: IEEE Computer Society, (2001), pp. 229-240.

[2] W. R. Davis, N. Zhang, K. CAMERA et Al., A Design Environment for High Throughput, Low Power Dedicated Signal Processing Systems. IEEE Journal of Solid-State Circuits, 37(3), (2002), pp. 420-431.

[3] P. Foglia, R. Giorgi, CA Prete, Evaluating Optimizations for Multiprocessors E-commerce Server Running TPC-W Workload, in Proceedings of the 34th Hawaii International Conference on System Sciences, Hawaii. Fourer, R. and J.P. Goux, 1, (2001), pp. 1-9.

[4] P. Foglia, R. Giorgi, C.A. Prete, A Simulation Study of Memory Performance of SMP Multiprocessors Running a TPC-W Workload. IEE Proceedings Computers and Digital Techniques (IEE CDT Sez. E), 151(2), (2004), pp. 93-109. 
[5] D. F. Garcia, J. Garcia, Tpc-w e-commerce benchmark evaluation. IEEE Computer, 36(2), (2003), pp. 42-48.

[6] F. J. HIRSCH, Introducing SSL and certificates using SSLeay. World Wide Web Journal, 2(2) (1997), pp. 141-173.

[7] D. A. MENASCÉ, TPC-W: A Benchmark for ECommerce, IEEE Internet Computing, 6(3) (2002), pp. 83-87.

[8] Database Management System DM4. http: //www.dameng.cn.

[9] Standard Performance Evaluation Corporation. SPECweb99 Benchmark. http://www.spec.org.

[10] SQL server Home. http://www.microsoft.com/sql/

[11] Transaction Processing Performance Council (TPC), TPC BENCHMARK ${ }^{T M} \mathrm{~W}$ (Web Commerce) Specification Version 1.8 http://www.tpc.org.

Received: May, 2005 Accepted: February, 2006

Contact address:

Zhu Hong

Huazhong University of Science and Technology

Wuhan, Hubei, 430074

P. R. China

e-mail: zhuhong@public.wh.hb.cn

Fu Xin

Huazhong University of Science and Technology Wuhan, Hubei, 430074

P. R. China

e-mail: fuxin@dameng.com

\author{
Lin Qiu Hu \\ Huazhong University of Science and Technology \\ Wuhan, Hubei, 430074 \\ P. R. China \\ e-mail: byzantine111@sohu.com \\ Kevin Lü \\ BBS, Room76, Tin Building \\ Brunel University \\ Uxbridge \\ UK UB8 3PH
}

e-mail: Kevin.Lu@brunel.ac.uk

ZHU HONG is an associate professor at the School of Computer Science and Technology, Huazhong University of Science and Technology, Wuhan, Hubei, China. She obtained her Ph.D. in Computer Software \& Theoretical Computer Science in 2001. Her current research areas are database security, database management system implementation.

FU XIN is an lecturer at the School of Computer Science and Technology, Huazhong University of Science and Technology, Wuhan, Hubei, China. He obtained his M.S. in Computer Software \& Theoretical Computer Science in 2002. His research areas include database management system implementation, software testing.

LIN QIU HUI is a software engineer in Oracle China Development Center. She obtained her M.S. in Computer Software \& Theoretical Computer Science in 2004. Her research areas are test of database system and performance evaluation.

KEVIN LÜ, PhD in Computer Science and BSc in Computer Science. $\mathrm{He}$ is a senior lecturer of business computing at Brunel University, UK. His current research areas of interests are data management, multi-agent system, intelligent data processing and enterprise information systems. 